\title{
Bringing circulating tumor DNA to the clinic in Hodgkin lymphoma
}

\section{David M. Kurtz}

Division of Oncology, Department of Medicine, Stanford University, Stanford, CA, USA.

E-mail: DAVID M. KURTZ - dkurtz@stanford.edu

doi:10.3324/haematol.2020.265165

O ver the last decade, technologies to detect, genotype, and track cancers non-invasively through the blood have been developed,${ }^{1.4}$ and are promising to revolutionize the way cancer is diagnosed and managed. These methods, termed "liquid biopsies", largely rely on the detection of tumor-derived cell-free DNA from the blood plasma of patients, or so-called circulating tumor DNA (ctDNA). More recently, research efforts have focused not only on the development of such methods, but on the translation into the clinic and on defining the utility of ctDNA in various malignancies. ${ }^{5.7}$ In this issue of Haematologica, Camus and colleagues ${ }^{8}$ explore the utility of ctDNA detection by an amplicon-based next-generation sequencing (NGS) approach in classical Hodgkin lymphoma ( $\mathrm{cHL}$ ) in a prospective observational study.

Detection of ctDNA largely has two major areas of clinic utility: i) genotyping of tumor-derived mutations non-invasively through the blood, and ii) disease quantification and detection of minimal residual disease (MRD) after therapy (Figure 1). In this article, the authors explore each of these potential uses in turn. They begin by assessing the performance of mutational genotyping from cell-free DNA in cHL, finding variants in the majority of cases (70\%). Importantly, in cases where both plasma and tumor DNA were available for sequencing, the authors found a high degree of concordance between mutations identified in the two compartments. Interestingly, they also found a higher median allele fraction in plasma than in tumor samples. This highlights a particular advantage of ctDNA for genotyping in cHL: due to the low abundance of malignant Reed-Sternberg (RS) cells, which are typically $\sim 1 \%$ or less of all cells in a tumor, genotyping and NGS from tumor samples in cHL is particularly difficult (Figure 1). This suggests that future studies elucidating the genetic landscape of $\mathrm{cHL}$ could benefit from studying cell-free DNA rather than tumor DNA. The excellent performance of mutational genotyping from cell-free DNA in this study confirms prior reports examining genotyping in cHL using targeted hybrid-capture sequencing from cell-free DNA., ${ }^{9,10}$ These results are in-line with the performance of ctDNA genotyping for other B-cell lymphomas, most notably diffuse large B-cell lymphoma (DLBCL), ${ }^{6,11,12}$ suggesting a potential broad utility for this assay in many lymphoma subtypes.

The authors go on to explore the utility of ctDNA quan-

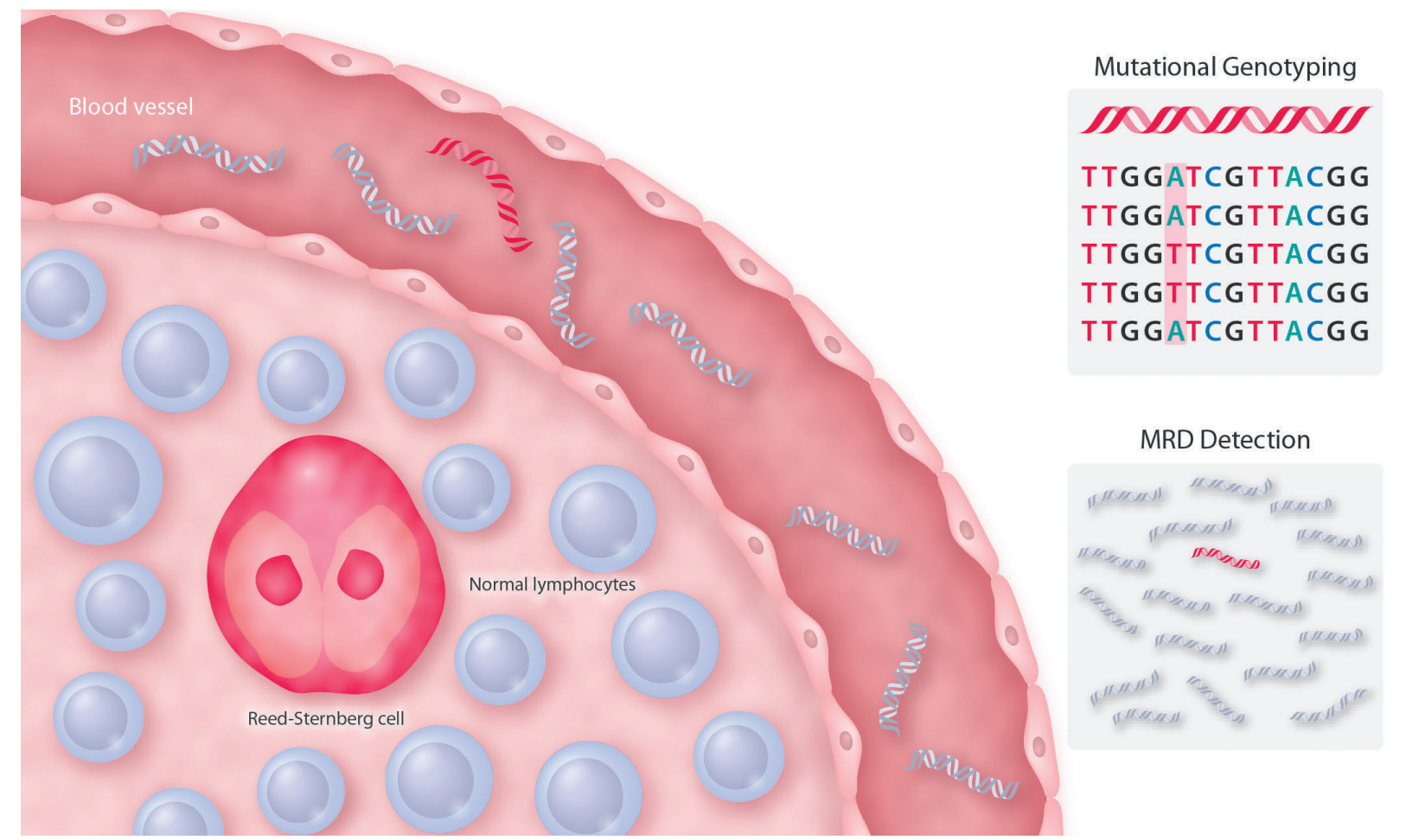

Figure 1. Circulating tumor DNA in Hodgkin lymphoma. This figure shows an example of the challenges and opportunities for circulating tumor DNA in classical Hodgkin lymphoma. In this disease, the malignant Reed-Sternberg cells represent only a small fraction of the cellular material in the tumor. DNA from these cells (red), along with normal DNA (grey), are released into the circulation and can be collected and sequenced. Sequencing cell-free DNA largely has two use cases. First, identification of tumor-derived somatic mutations (top right), where cell-free DNA potentially contains a higher concentration of mutated molecules than DNA isolated from the tumor. Second, detection of low level minimal residual disease (MRD) during or after therapy (bottom right), where high sensitivity is critically important. 
titation and MRD using their assay. When quantifying pretreatment ctDNA, they observed a number of striking correlations, including higher stage and International Prognostic Score (IPS) with higher ctDNA concentrations. Higher ctDNA was also correlated with higher tumor volume measured by positron emission tomography/computerized tomography (PET/CT) scans. Again, these results are remarkably consistent with results from other lymphomas including DLBCL,,${ }^{6,13}$ where higher stage, International Prognostic Index (IPI), and tumor volumes are associated with high ctDNA. The authors finally go on to explore the utility of their assay for the detection of MRD after two cycles of therapy. Interestingly, no patients had detectable $M R D$ at this landmark, including patients who had eventual disease progression. While this suggests a high specificity of the assay, it also suggests improvements in sensitivity might be needed. Previous reports of ctDNA monitoring during therapy for $\mathrm{cHL}$ have suggested patients with suboptimal treatment response by PET scans (i.e., not in a complete response on interim imaging) can have residual detectable ctDNA; $;, 10$ however, these studies have a limited number of cases. These data do suggest that sensitivity matters for detection of low-burden disease - particularly during timepoints of radiographic response and remission. The performance of sensitive ctDNA assays for detection of MRD and molecular response in CHL, DLBCL, ${ }^{13}$ and other lymphomas should be validated in additional large prospective studies. It is likely that additional technical improvements in sensitivity will also improve the performance in these low disease burden use cases.

While this report from Camus and colleagues, as well as reports from other groups, confirms the utility of ctDNA for mutational genotyping and disease detection in $\mathrm{cHL}$ and other lymphomas, a number of technical and logistical hurdles remain. While ctDNA-based mutational genotyping has shown utility for identifying single-nucleotide variants, other types of somatic alterations are more challenging to identify from plasma DNA, including copy number alterations, small insertions and deletions, and structural variants. New methods to detect these types of variants are therefore needed. Additionally, with multiple methods including amplicon and hybrid-capture based approaches finding their way into clinical laboratories, discrepancies in methodology and test characteristics are becoming more apparent, making comparisons between tests difficult. Efforts to harmonize and standardize ctDNA quantification and reporting are therefore needed for translation into the clinic. Furthermore, larger prospective studies are required to put the clinical utility of ctDNA quantification as a prognostic marker into context through multivariable analyses considering metabolic tumor volume and clinical risk factors such as the IPS for $\mathrm{cHL}$ and the IPI for DLBCL.

Finally, and perhaps most significantly, questions remain regarding how to act on ctDNA assessments. While high pretreatment ctDNA level and failure to achieve a molecular response are markers of patients with a high risk of treatment failure, how to improve outcomes for these patients remains unclear. PET-adapted approaches have become common in $\mathrm{cHL} ; ;^{14,15}$ however, these approaches have been difficult to implement for DLBCL, potentially due to the imperfect sensitivity and specificity in this dis- ease. Similar risk-adapted trial designs in both $\mathrm{cHL}$ and DLBCL using ctDNA molecular response to guide therapy will therefore be needed to demonstrate superior outcomes and ultimately lead ctDNA assessment into routine clinical care. Finally, further research is needed to establish the utility of ctDNA in other lymphoma subtypes, particularly in low-grade lymphomas where clinical management strategies typically do not aim for curative endpoints. There still is therefore significant work ahead in translation of liquid biopsies into routine clinical management of B-cell lymphomas; however, early data from studies such as the work from Camus and colleagues provide promising evidence that ctDNA will change clinical management for B-cell lymphomas in the not-too-distant future.

\section{Disclosures}

Dr. Kurtz has served as a consultant for Roche and Genentech, has ownership equity in Foresight Diagnostics, and has patents pending related to methods for analysis of cell free nucleic acids and methods for treatment selection based on statistical frameworks of clinical outcome.

\section{Contributions}

$D M K$ conceived of and wrote this manuscript.

\section{References}

1. Bettegowda C, Sausen M, Leary RJ, et al. Detection of circulating tumor DNA in early- and late-stage human malignancies. Sci Trans Med. 2014;6(224):224ra24.

2. Diehl F, Schmidt K, Choti MA, et al. Circulating mutant DNA to assess tumor dynamics. Nat Med. 2008;14(9):985-990.

3. Newman AM, Bratman SV, To J, et al. An ultrasensitive method for quantitating circulating tumor DNA with broad patient coverage. Nat Med. 2014;20(5):548-554.

4. Newman AM, Lovejoy AF, Klass DM, et al. Integrated digital error suppression for improved detection of circulating tumor DNA. Nat Biotechnol. 2016;34(5):547-555

5. Abbosh C, Birkbak NJ, Wilson GA, et al. Phylogenetic ctDNA analysis depicts early-stage lung cancer evolution. Nature. 2017;545(7655):446451.

6. Scherer F, Kurtz DM, Newman AM, et al. Distinct biological subtypes and patterns of genome evolution in lymphoma revealed by circulating tumor DNA. Sci Transl Med. 2016;8(364):364ra155.

7. Tie J, Wang Y, Tomasetti C, et al. Circulating tumor DNA analysis detects minimal residual disease and predicts recurrence in patients with stage II colon cancer. Sci Transl Med. 2016;8(346):346ra92.

8. Camus V, Viennot M, Lequesne J, et al. Targeted genotyping of circulating tumor DNA for classical Hodgkin Lymphoma monitoring: a prospective study. Haematologica. 2020;106(1):154-162.

9. Desch AK, Hartung K, Botzen A, et al. Genotyping circulating tumor DNA of pediatric Hodgkin lymphoma. Leukemia. 2020;34(1):151-166.

10. Spina V, Bruscaggin A, Cuccaro A, et al. Circulating tumor DNA reveals genetics, clonal evolution, and residual disease in classical Hodgkin lymphoma. Blood. 2018;131(22):2413-2425.

11. Bohers E, Viailly PJ, Becker S, et al. Non-invasive monitoring of diffuse large B-cell lymphoma by cell-free DNA high-throughput targeted sequencing: analysis of a prospective cohort. Blood Cancer J. 2018;8(8):74.

12. Rossi D, Diop F, Spaccarotella E, et al. Diffuse large B-cell lymphoma genotyping on the liquid biopsy. Blood. 2017;129(14):1947-1957

13. Kurtz DM, Scherer F, Jin MC, et al. Circulating tumor DNA measurements as early outcome predictors in diffuse large B-cell lymphoma. J Clin Oncol. 2018;36(28):2845-2853.

14. Andre MPE, Girinsky T, Federico M, et al. Early positron emission tomography response-adapted treatment in stage I and II Hodgkin lymphoma: final results of the randomized EORTC/LYSA/FIL H10 trial. J Clin Oncol. 2017;35(16):1786-1794.

15. Johnson P, Federico M, Kirkwood A, et al. Adapted treatment guided by Interim PET-CT scan in advanced Hodgkin's lymphoma. N Engl J Med. 2016;374(25):2419-2429. 